\title{
High-frequency vortex ratchet effect in a superconducting film with a nanoengineered array of asymmetric pinning sites
}

\author{
B. B. Jin, ${ }^{1,2}$ B. Y. Zhu, ${ }^{3}$ R. Wördenweber, ${ }^{4}$ C. C. de Souza Silva, ${ }^{5}$ P. H. Wu, ${ }^{2}$ and V. V. Moshchalkov ${ }^{1}$ \\ ${ }^{1}$ Institute for Nanoscale Physics and Chemistry (INPAC), Katholieke Universiteit Leuven, Celestijnenlaan 200D, B-3001 Leuven, Belgium \\ ${ }^{2}$ Research Institute of Superconductor Electronics (RISE), School of Electronic Science and Engineering, Nanjing University, \\ Nanjing 210093, China \\ ${ }^{3}$ National Laboratory for Superconductivity, Institute of Physics, Beijing National Laboratory for Condensed Matter Physics, \\ Chinese Academy of Sciences, Beijing 100190, China \\ ${ }^{4}$ Institut für Bio-und Nanosysteme, Bioelektronik (IBN-2), Foschungszentrum Jülich, D-52425 Jülich, Germany \\ ${ }^{5}$ Departamento de Física, Universidade Federal de Pernambuco, 50670-901 Recife, PE, Brazil \\ (Received 23 October 2009; revised manuscript received 22 March 2010; published 5 May 2010)
}

\begin{abstract}
Vortex ratchet effect is investigated experimentally in the frequency range between $0.5 \mathrm{MHz}$ and $2 \mathrm{GHz}$. The ratchet potential is provided by an array of about a quarter of a million nanoengineered asymmetric antidots in $\mathrm{a} \mathrm{Pb}$ film. A square vortex lattice is stabilized at the first matching field, when each asymmetric antidot is occupied by a single vortex. We have found that (1) the transition from adiabatic to nonadiabatic cases occurring at about $1 \mathrm{MHz}$, above which the ratchet windows shift upwards with the applied frequency due to the fact that the time for a vortex to escape from the pinning potential is comparable to the period of the applied rf driving current $I_{\mathrm{rf}}$; (2) a sudden $V_{\mathrm{dc}}$ reversal at large $I_{\mathrm{rf}}$, which can be attributed to inertia effect; (3) the collective step-motor behavior in the $\mathrm{MHz}$ region, i.e., the vortex lattice moves forward by an integer number of the period of pinning array at each cycle of $I_{\mathrm{rf}}$; and (4) very weak ratchet effect at several $\mathrm{GHz}$, indicating the possibility of stronger inertia effects in the vortex motion at such high frequencies. These results reveal rich physics information in the nonadiabatic ratchet system and are of particular importance for particle separation and molecular motor in biology.
\end{abstract}

DOI: 10.1103/PhysRevB.81.174505

PACS number(s): 85.25.-j, 74.78.Na, 74.25.- q, 05.40.-a

\section{INTRODUCTION}

Ratchet is a system where particles can acquire a net motion in an asymmetric periodic potential in the presence of deterministic or stochastic forces with time averages of zero. ${ }^{1,2}$ When the forces are spatially uniform, time periodic, and deterministic, we have the so-called rocking ratchet. ${ }^{3-5}$ This kind of ratchet has attracted great attention during the past 20 years, and a variety of new physical phenomena have been revealed. ${ }^{4,5}$

Vortex array in superconductors has been proven to be a nice system to study the rocking ratchet effect. ${ }^{6-26}$ Either an array of triangular magnetic dots or an array of asymmetric antidotes can serve as pinning sites to trap the quantized flux lines to form a vortex array. ${ }^{10-21}$ With a periodic and asymmetrical pinning potential, this vortex array rectifies the applied alternative current to produce dc voltage output $V_{\mathrm{dc}}$, just as what a diode does. The advantages of such a system are the following. (1) By nature it is a two-dimensional (2D) multiparticle ratchet system, which contains a large amount of the vortex cells with long-range interactions between them, making it possible to observe rich physical phenomena here $^{12,13}$ which are different those in the case of single particle in a one-dimensional potential. For example, simulations demonstrate that molecular motors can cooperate in large groups, and the combination of a large number of interacting particles will lead to ineluctable dynamical phase transition and instabilities, ${ }^{27,28}$ (2) strong rectification can be expected because the voltage is proportional to the number of the vortices when they move in a coherent way; and (3), the asymmetric potential can be tuned by changing the size and shape of the pinning sites, as well as the polarity of the vortices in the magnetic dot array. Based on such an asymmetric system, one can study experimentally the inertia effect, ${ }^{15,16}$ the controllable multiple reversals of $V_{\mathrm{dc}}{ }^{17}$ the reversed vortex ratchet, ${ }^{20}$ predict numerically the quantization of $V_{\mathrm{dc}},{ }^{13}$ and new vortex configurations as a function of vortex density. ${ }^{7}$

Up to now, in vortex ratchet measurements the frequencies of the driving ac current are always lower than 1 MHz. ${ }^{14-20}$ In the low-frequency regime, the vortex in one temporal period can pass through a very large number of the asymmetric pinning wells. As a consequence, the rectified voltage output does not depend on the frequency, which corresponds to the adiabatic limit. In particular, it is difficult to observe experimentally the quantization of $V_{\mathrm{dc}}$ or phase locking between the rf driving and the vortex motion. ${ }^{3}$ According to theoretical calculation, ${ }^{13} V_{\mathrm{dc}}$ vs the amplitude of the applied driving current $I_{\mathrm{rf}}$ shows equidistant steps, which occur at $n B_{1} L d_{0} f$, where $n$ is the number of the step, $B_{1}$ is the first matching field, $L$ is the distance between two voltage contacts, $d_{0}$ is the lattice constant of vortex array and $\mathrm{f}$ is the working frequency. In the present experiments, ${ }^{15}$ we choose $L=2 \mathrm{~mm}$ and the spacing $d_{0}$ of the periodic pinning arrays is $1.5 \mu \mathrm{m}$, which correspond to $B_{1}=0.92 \mathrm{mT}$. So, $V_{\mathrm{dc}}$ is 2.76 $\mathrm{nf}$, where $V_{\mathrm{dc}}$ and $\mathrm{f}$ are in the units of $\mu \mathrm{V}$ and $\mathrm{MHz}$, respectively. In order to achieve the significant rectification output and the distinguished step structure in the voltage versus the ac current amplitude, the applied frequency should be larger than several $\mathrm{MHz}$; in other words, the available frequency should be at least in $\mathrm{MHz}$ range for investigating the nonadiabatic ratchet effect. 

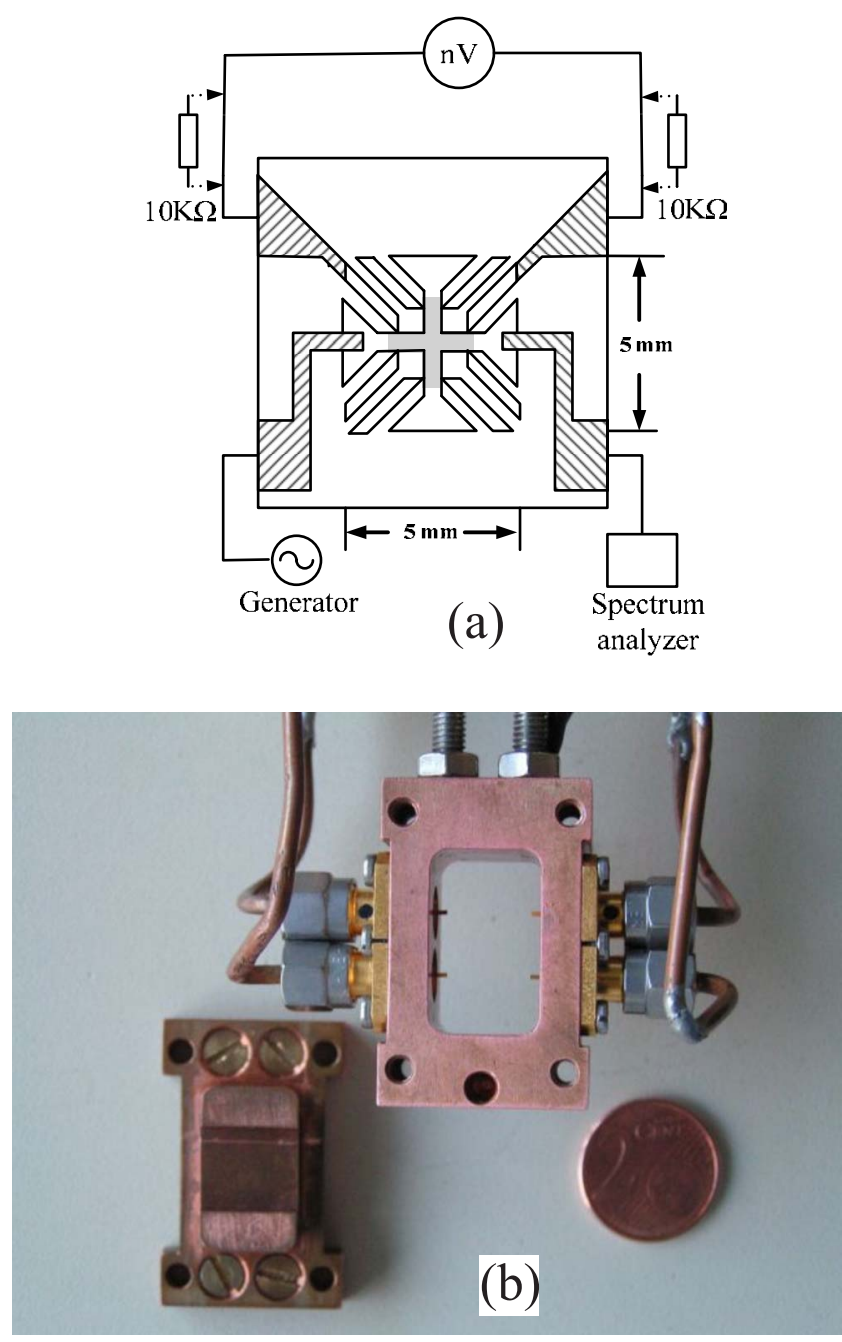

FIG. 1. (Color online) (a) The schematic illustration of the sample and the measurement setup; (b) the photograph of samplemounted copper box.

In the present work, with ac currents at frequencies above $\mathrm{MHz}$, we study the transport of vortices in superconducting thin-film samples containing regular arrays of asymmetric pinning wells. The main purpose is to observe the phase locking phenomena, which is very challenging but most desirable in the field of superconducting vortex ratchet. In the frequency range from $0.5 \mathrm{MHz}$ to $2 \mathrm{GHz}$, we measure $V_{\mathrm{dc}}$ vs $I_{\mathrm{rf}}$, or input power $P_{\mathrm{in}}$. The results below $100 \mathrm{MHz}$ are elaborately studied. The depinning current is found to be almost constant below $1 \mathrm{MHz}$, and increases with the rf current above $1 \mathrm{MHz}$. Our results are quite different from the observations in the $\mathrm{KHz}$ region, and a pronounced phase transition in the vortex ratchet from the adiabatic process to nonadiabatic process is demonstrated. Most importantly, in the curves of $V_{\mathrm{dc}}$ vs $I_{\mathrm{rf}}$, the voltage steps caused by phase locking are obtained in the $\mathrm{MHz}$ frequency range, while in the $\mathrm{GHz}$ frequency range the ratchet effect becomes very weak indicating the high-frequency limitation for the rectification in our ratchet system.

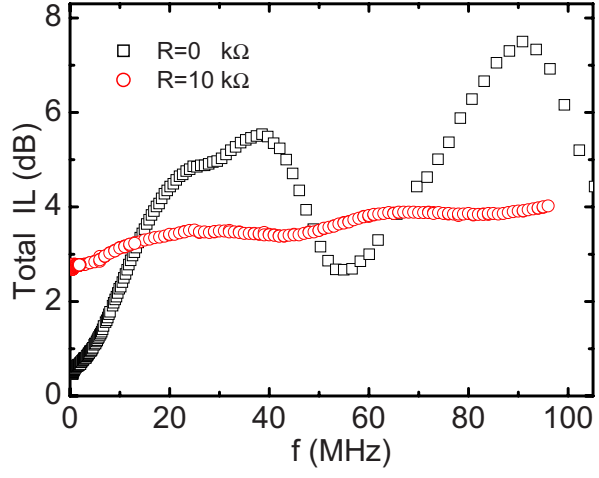

FIG. 2. (Color online) Total transmission loss versus the frequency without (black square) and with resistors (red circle) in the voltage branches.

\section{SETUP AND SAMPLE PREPARATION}

The sample and measurement setup are schematically shown in Fig. 1(a). A 50-nm-thick superconducting $\mathrm{Pb}$ film $\left(T_{\mathrm{c}}=7.15 \mathrm{~K}\right)$ in a cross-shaped geometry $(5 \mathrm{~mm} \times 5 \mathrm{~mm})$ is deposited on $\mathrm{SiO}_{2}$ substrate. ${ }^{15,29}$ The readers are referred to Ref. 29 for the details of film deposition and lift-off procedures. As indicated by the shaded area in Fig. 1(a), horizontally there is a nanoengineered array of antidotes, $2.0 \mathrm{~mm}$ long and $0.3 \mathrm{~mm}$ wide, in which each antidote is made of two adjacent square holes so that an asymmetric double-well pinning potential is created when the driving current has a horizontal component. ${ }^{15,26}$ As the period in the array is $1.5 \mu \mathrm{m}$, corresponding to the first matching field of 0.92 $\mathrm{mT}$, there are altogether $1300 \times 200$ antidots in it roughly. Thus a quarter of a million individual quantum vortices are pinned when the applied magnetic field is equal to the first matching field. The hatched parts in Fig. 1(a) are four gold contacts, deposited using a metallic mask, to make the electric connections easy during the measurements. Finally, the sample was inserted into the sample-mounted copper box shown in Fig. 1(b), and the pins of coaxial rf connectors attached on the gold layer to form a nice contact. The transport measurements are performed in a flow-helium cryostat. As shown in Fig. 1(a), the rf current, at $250 \mathrm{KHz}$ to $4 \mathrm{GHz}$ and with a power range of -10 to $15 \mathrm{dBm}$, is applied to the sample via a grounded coaxial cable and the rf current path is closed by a spectrum analyzer which monitors and records the power. The dc voltage output across the sample is measured by a nanovoltmeter. A Helmholtz coil is used to produce the required magnetic field perpendicular to the sample surface.

The transmission property of this system is examined before the ratchet measurements are made. The total insertion loss (IL), which is the ratio (expressed in $\mathrm{dB}$ ) of the input power from the source to the power received by the spectrum analyzer, is measured at $7 \mathrm{~K}$ from $250 \mathrm{KHz}$ to $100 \mathrm{MHz}$ and without applied magnetic field. This loss has a large ripple (Fig. 2) over the designated frequency range. In order to understand the origin of the ripple and to find a way to reduce it, we note that the present arrangement is in fact a four-probe one; the current goes from the generator to the spectrum analyzer via the "current arm," while a nanovolt- 


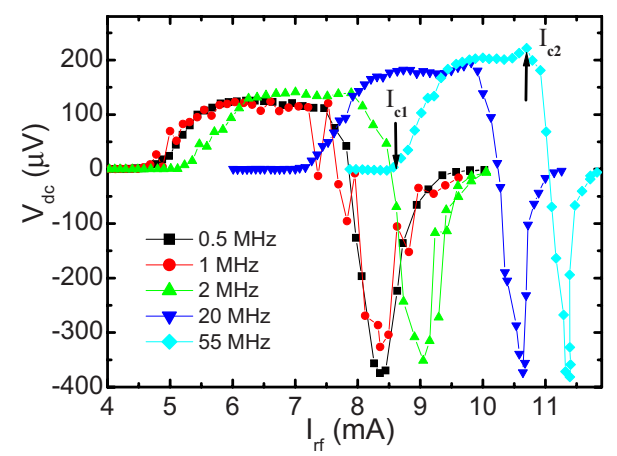

FIG. 3. (Color online) The rectified dc voltage $V_{\mathrm{dc}}$ dependence on the input rf current $I_{\mathrm{rf}}$ at $0.5,1,2,20$, and $50 \mathrm{MHz} . I_{\mathrm{c} 1}$ and $I_{\mathrm{c} 2}$ represent two critical currents where $V_{\mathrm{dc}}$ occurs and reverses. The shift of $I_{\mathrm{c} 1}$ and $I_{\mathrm{c} 2}$ is clearly seen from the figure.

meter is used to measure the voltage across the sample in the "voltage arm." At low frequencies there is little coupling between the current arm and the voltage arm. At high frequencies, however, rf current has many ways to go. For example, it can not only flow through the current arm but also be coupled to the voltage arm where it can get reflected by the nanovoltmeter and return to the current path. Such additional paths behave like some transmission lines in shunt with the current arm, and the shunting circuits can resonate at some specific frequencies (here, at about 40 and $90 \mathrm{MHz}$ ), absorbing the rf current and causing the loss to increase. To reduce this ripple, we insert two $10 \mathrm{k} \Omega$ resistors into the voltage arm as shown in Fig. 1(a). The resistors damp the resonance, giving rise to a relatively flat frequency response (circle symbols in Fig. 2).

\section{EXPERIMENTAL RESULTS AND DISCUSSIONS}

In this section we are going to present our experimental results on four subjects covering the transition from adiabatic to nonadiabatic processes, $V_{\mathrm{dc}}$ reversal at large $I_{\mathrm{rf}}$, the collective step-motor behavior, and the ratchet effect at GHz. The experimental results are described in details, discussed thoroughly, and often accompanied by theoretical calculations.

\section{A. Transition from adiabatic to nonadiabatic cases}

With an input power of $0 \mathrm{dBm}$ at $0.5 \mathrm{MHz}$, we first measure the dependence of the rectified dc voltage $V_{\mathrm{dc}}$ on the magnetic field $H$ at $7 \mathrm{~K}$. A clear sign inversion of $V_{\mathrm{dc}}$ is observed as $H$ changes its sign. In addition, an almost symmetrical behavior is obtained when the remnant field effect is taken into account. These results demonstrate unambiguously that $V_{\mathrm{dc}}$ comes from the intrinsic rectifying properties of the asymmetrical antidot configurations.

Figure 3 shows $V_{\mathrm{dc}}$ at $0.5,1,2,20$, and $55 \mathrm{MHz}$ as a function of $I_{\mathrm{rf}}$ at $7 \mathrm{~K}$ and $B_{1} . I_{\mathrm{rf}} \mathrm{s}$ are calculated from the transmitted power indicated on the spectrum analyzer and its input impedance $50 \Omega$. At low $I_{\mathrm{rf}}, V_{\mathrm{dc}}$ is zero because all vortices are pinned. When $I_{\mathrm{rf}}$ increases and exceeds the first critical current value $I_{\mathrm{c} 1}$ of the asymmetric pinning potential, a finite $V_{\mathrm{dc}}$ appears and increases with $I_{\mathrm{rf}}$. Then, $V_{\mathrm{dc}}$ starts to

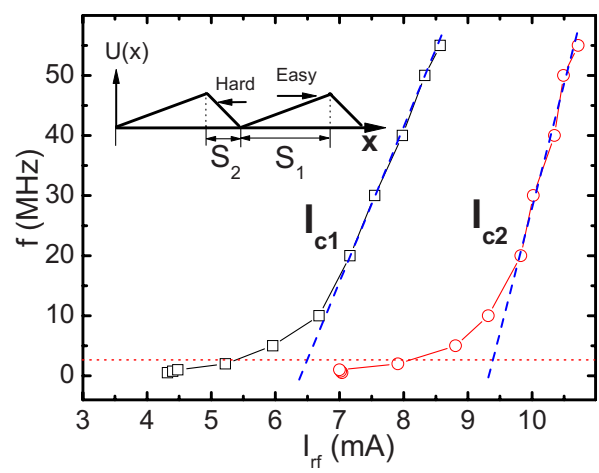

FIG. 4. (Color online) The frequency dependence of the $I_{\mathrm{c} 1}$ and $I_{\mathrm{c} 2}$ for the asymmetric pinning potentials. The blue dash lines represent the fit to the results at and above $20 \mathrm{MHz}$. The horizontal red dot line represents a rough separation between low and highfrequency regimes.

decrease when the second critical current $I_{\mathrm{c} 2}$ is reached, followed by a sudden reversal, and $V_{\mathrm{dc}}$ comes back to zero at extremely large $I_{\mathrm{rf}}$. This voltage reversal appears in all our measurements $(0.5 \mathrm{MHz}-2 \mathrm{GHz})$ in a similar way.

Three features can be found from the above results. The first one is that the dependence of $V_{\mathrm{dc}}$ on $I_{\mathrm{rf}}$ at 0.5 and $1 \mathrm{MHz}$ are almost the same but quite different from those at other frequencies above $1 \mathrm{MHz},{ }^{15}$ indicating the ratchet effect strongly depends on frequency. This difference can be attributed to the transition from the adiabatic case to the nonadiabatic case, or from the low-frequency $(<1 \mathrm{MHz})$ region to the high-frequency $(>1 \mathrm{MHz})$ region, and the characteristic frequency is about $1 \mathrm{MHz}$ in our ratchet system. The second one is that both $I_{\mathrm{c} 1}$ and $I_{\mathrm{c} 2}$ shift upwardly with the increase of the measurement frequencies, which is quite different from the reports at low frequencies. The third one is the reversal of $V_{\mathrm{dc}}$ at large $I_{\mathrm{rf}}$, which will be discussed in detail in another subsection.

With regard to the shift of $I_{\mathrm{c} 1}$ and $I_{\mathrm{c} 2}$, we know from previous analyses that in the low-frequency region they represent the depinning currents along the "weak" and "strong" directions, respectively, for the asymmetrical potential determined by the composite antidote array; and they should be frequency independent. In our measurements as shown in Fig. $4, I_{\mathrm{c} 1}$ and $I_{\mathrm{c} 2}$ are almost constant below $1 \mathrm{MHz}$. However, as the driving frequency goes into several $\mathrm{MHz}, I_{\mathrm{c} 1}$ and $I_{\mathrm{c} 2}$ increase with the frequency, and follow a linear dependence for frequencies above $20 \mathrm{MHz}$. These shifts of $I_{\mathrm{c} 1}$ and $I_{\mathrm{c} 2}$ can be due to the finite time needed for vortices to escape the pinning potential well. In the high-frequency regime, the drive period may be too short for vortices to be depinned in the positive half cycle. The vortices are then pulled back to the starting point during the negative cycle, and no net dc motion occurs. So, a higher driving current is needed for the appearance of a nontrivial dc voltage response, leading to the increase of $I_{\mathrm{c} 1}$ with the frequency. Similarly for $I_{\mathrm{c} 2}$, a higher driving current makes the vortices to overcome the pinning potential along the strong direction to reduce $V_{\mathrm{dc}}$.

To further analyze this shift, we assume that the asymmetric potential profile has a saw tooth shape as shown in the inset of Fig. 4 where $S_{1}$ is the distance for the potential to get 
to its extreme while $S_{2}$ is the distance for it to go back to zero. This simple profile has been proven to be useful for studying the ratchet effect. Then, the motion of a vortex in the overdamped approximation is described by

$$
\eta V=F_{\mathrm{L}}+F_{\mathrm{vv}}+F_{\mathrm{p}}
$$

where $\eta$ is the viscous drag coefficient. $V$ is the velocity of the vortex. $F_{\mathrm{L}}$ is the Lorentz force induced by the applied current. $F_{\mathrm{vv}}$ is the repulsive vortex-vortex interaction, which cancels each other in our case since the applied magnetic field equals to the first matching field, and $F_{\mathrm{p}}$ is the pinning force determined by the antidots in the film. Here, we assume $F_{\mathrm{L}}$ and $F_{\mathrm{p}}$ are respectively proportional to the applied current $I_{1} \sin (2 \pi f t)$ and $I_{\mathrm{c} 01}$, the depinning current in the dc case and along the easy direction, i.e., $F_{\mathrm{L}}=A I_{1} \sin (2 \pi f t)$ and $F_{\mathrm{p}}=A I_{\mathrm{c} 01}$, where $A$ and $f$ are the amplitude and frequency of the driving force, respectively. To overcome the potential barrier and move to the next potential well, the vortex should move at least a distance of $S_{1}$ in half cycle. So, if we consider a driving current $I_{\mathrm{c} 1}(f)$, we may have

$$
\frac{A}{\eta} \int_{0}^{\mathrm{T} / 2}\left(I_{\mathrm{c} 1} \sin (\omega t)-I_{\mathrm{c} 01}\right) d t=S_{1}
$$

After integration, we can obtain

$$
I_{\mathrm{c} 1}=\frac{\pi \eta S_{1}}{A} f+\frac{\pi}{2} I_{\mathrm{c} 01} .
$$

Similarly,

$$
I_{\mathrm{c} 2}=\frac{\pi \eta S_{2}}{A} f+\frac{\pi}{2} I_{\mathrm{c} 02} .
$$

In Eqs. (3) and (4), we can see that both $I_{\mathrm{c} 1}$ and $I_{\mathrm{c} 2}$ depend on the driving frequency linearly. We may argue that with the increase of the frequency, the period becomes very short, which results in extremely strong driving force for effectively rectifying the vortex motion in the periodic array of asymmetric pinning wells. In this case, critical currents $I_{\mathrm{c} 01}$ and $I_{\mathrm{c} 02}$ for the vortex pinning in both weak and strong directions do not dominate the rectification window any more. Furthermore the values of $I_{\mathrm{c} 1}$ and $I_{\mathrm{c} 2}$, above which their frequency dependences become linear, are about 6.3 and 9.3 $\mathrm{mA}$, respectively. If we assume that the measured $I_{\mathrm{c} 1}$ and $I_{\mathrm{c} 2}$ below $1 \mathrm{MHz}$ are $I_{\mathrm{c} 01}(4.4 \mathrm{~mA})$ and $I_{\mathrm{c} 01}(7.0 \mathrm{~mA})$, the ratios of $I_{\mathrm{c} 1} / I_{\mathrm{c} 01}$ and $I_{\mathrm{c} 2} / I_{\mathrm{c} 02}$ are about 1.43 and 1.33 , which are close to 1.57 predicted from Eqs. (3) and (4). The relative small ratio of $I_{\mathrm{c} 2} / I_{\mathrm{c} 02}$ may be caused by thermal effect. Both $I_{\mathrm{c} 1}$ and $I_{\mathrm{c} 2}$ heat the sample, raise its temperature, and reduce $I_{\mathrm{c} 01}$ or $I_{\mathrm{c} 02}$. But as $I_{\mathrm{c} 2}$ is grater than $I_{\mathrm{c} 1}$, it has a stronger effect than $I_{\mathrm{c} 1}$, causing a larger reduction in $I_{\mathrm{c} 02}$ than that in $I_{\mathrm{c} 01}$. So, the above value of $I_{\mathrm{c} 2} / I_{\mathrm{c} 02}$ is overestimated. Therefore, the frequency dependence of $I_{\mathrm{c} 1}$ and $I_{\mathrm{c} 2}$ not only gives further evidence that our data (Fig. 3) originate from the vortex rectification by the composite pinning well array in the superconducting film, but also demonstrates the transition process from low-frequency to high-frequency regimes.

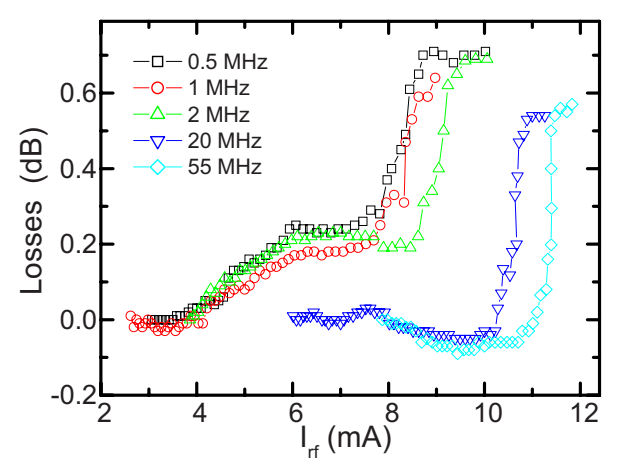

FIG. 5. (Color online) The loss during the rectification as a function of $I_{\mathrm{rf}}$ at $0.5,1,2,20$, and $50 \mathrm{MHz}$, respectively.

\section{B. Sudden $V_{\mathrm{dc}}$ reversal at large $\boldsymbol{I}_{\mathrm{rf}}$}

We address in this subsection the sudden reversal of $V_{\mathrm{dc}}$ at large $I_{\mathrm{rf}}$ shown in Fig. 3. To get insight into the mechanism of this reversal, the losses during the rectification, i.e., $10 \log \left[P_{\text {rec }}\left(I_{\mathrm{rf}}\right) / P_{\text {rec }}\left(I_{\mathrm{rf}} \ll I_{\mathrm{c} 1}\right)\right]$, are depicted in Fig. 5 where $P_{\text {rec }}$ is the power received at the spectrum analyzer. It is clearly seen that the $V_{\mathrm{dc}}$ reversal in Fig. 3 appears in the same current region where the losses sharply increase as seen in Fig. 5. This increase is about $0.5 \mathrm{dBm}$. We also measure the temperature dependence of $P_{\text {rec }}$ at $0.5 \mathrm{MHz}$ as seen in Fig. 6 with input power of $-10 \mathrm{dBm}$ and $B=0$. The similar abrupt change for $P_{\text {rec }}$ is observed when the transition from superconducting to normal states occurs. So, this reversal is proposed to be related to superconducting transition. The size of the vortex becomes large $\left(\xi \rightarrow \infty\right.$ as $\left.T \rightarrow T_{\mathrm{c}}\right)$ in this voltage reversal region, which is equivalent to the increase of the vortex mass and which leads to strong inertial effect at large driving amplitudes. ${ }^{30,31}$

Now let us consider the 2D problem of a lattice of inertial vortices interacting with a $2 \mathrm{D}$ array of asymmetric pinning sites, which can be modeled as two superposed square sublattices of strong and weak attractive potential. ${ }^{12,13,15,16}$ The sublattices have a spacing $d_{0}(=1$ here $)$ and are shifted with respect to each other by a distance $0.36 d_{0}$, which is the same as the separation in the experimental sample. ${ }^{15}$ All pinning centers are modeled by Gaussian potential wells with a decay length $R_{\mathrm{p}}$. The pinning force exerted by the $k$ th pin of one sublattice on the $i$ th vortex is

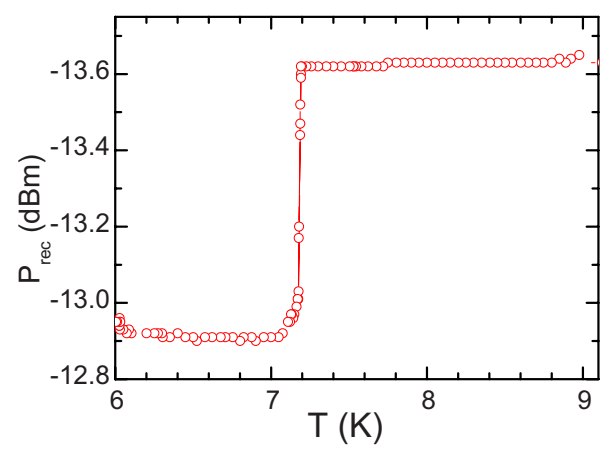

FIG. 6. (Color online) The temperature dependence of the received power by the spectrum analyzer. Here, the measured frequency is about $0.5 \mathrm{MHz}$ with the input power of $-10 \mathrm{dBm}$ and $B=0$. 


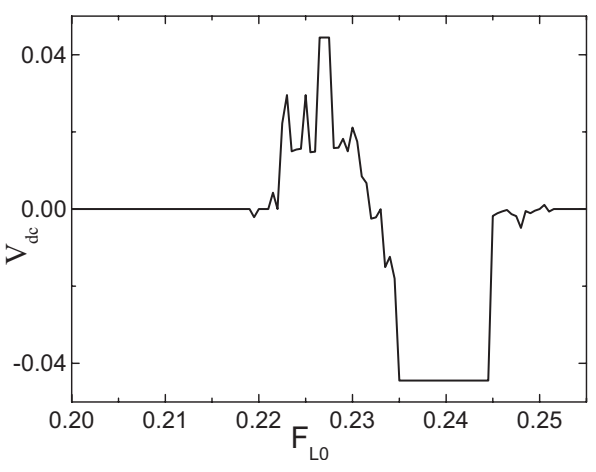

FIG. 7. Simulation result of $V_{\mathrm{dc}}$ dependence on the ac drive amplitude. Here the period $\mathrm{P}$ of the ac drive is $2250 \tau_{0}$ and $M=1$.

$$
F_{\mathrm{p}}^{s, w}\left(\mathbf{r}_{i}\right)=-F_{\mathrm{p} 0}^{s, w} \frac{\mathbf{r}_{i}-R_{k}^{s, w}}{R_{\mathrm{p}}} \exp \left(-\left|\frac{\mathbf{r}_{i}-R_{k}^{s, w}}{R_{\mathrm{p}}}\right|^{2}\right),
$$

where $\mathbf{r}_{i}$ represents the location of the $i$ th vortex and $\mathbf{R}_{k}^{s, w}$ is the location of the $k$ th pin of the weak $(w)$ and the strong $(s)$ sublattice, respectively. The intensity of the individual pinning force is denoted by $F_{\mathrm{p} 0}^{s, w} f_{0}$. We define the ratio between the weak and strong pinning strength as $\epsilon=F_{\mathrm{p} 0}^{w} / F_{\mathrm{p} 0}^{s}$. In our simulation, forces are measured in units of $f_{0}=\Phi_{0}^{2} / 8 \pi^{2} \Lambda^{3}$, with $\Lambda$ the effective superconducting penetration depth. The ac sine driving force can be modeled as $\mathbf{F}_{\mathrm{L}}(t)=\mathbf{F}_{\mathrm{L} 0} \sin (\omega t)$, where $\omega=2 \pi / P$ and $P$ is the period of the ac drive. Since we focus on the first matching field in our studies, the vortexvortex interaction can be completely cancelled out in the system. Then we may neglect the vortex-vortex interaction term in our simulation equation, and the underdamped equation of motion for an individual vortex is given by

$$
M \dot{\mathbf{v}}=\mathbf{F}_{\mathrm{L}}+\mathbf{F}_{\mathrm{p}}-\eta \mathbf{v} .
$$

Parameters used in the simulations are $R_{\mathrm{p}}=0.13, F_{\mathrm{p} 0}^{s}=0.5$, and $\epsilon=0.925$. The discrete time step is $\tau_{0}=0.01 . M$ is the effective mass of an individual vortex. The other parameters are similar to those in Ref. 16. In order to mimic the nonadiabatic limit of the extremely high-frequency ratchet, we choose the viscosity to be $\eta=1$ and, for simplicity, the effective mass of the individual vortex to be $M=1$ in our simulations. When the ac driving force is strong enough to assist the vortices to overcome the strong pinning at $I_{\mathrm{c} 2}$, a sharp jump of the effective mass of the individual vortices is reasonable for the critical transition. We confirm that the heavy vortex assumption and the dependence on the driving strength are indispensable, for the negative dc response of the extremely high ratchet effect in our molecular-dynamics simulations. In Fig. 7, we show the results of the simulations at a relatively short excitation period, and the sign reversal of the $V_{\mathrm{dc}}$ in the high drive region can be reproduced very well. Here, we would like to point out that we confine ourselves to the case where $H_{\mathrm{dc}}$ is equal to the first matching field of our system, which means no more than one vortex can be trapped in one ratchet unit cell and there is almost no interstitial vortex in the system. This is completely different from the case of multitrapped vortex system, ${ }^{17,26}$ where many vortices can be trapped in one ratchet unit cell. In the multi-

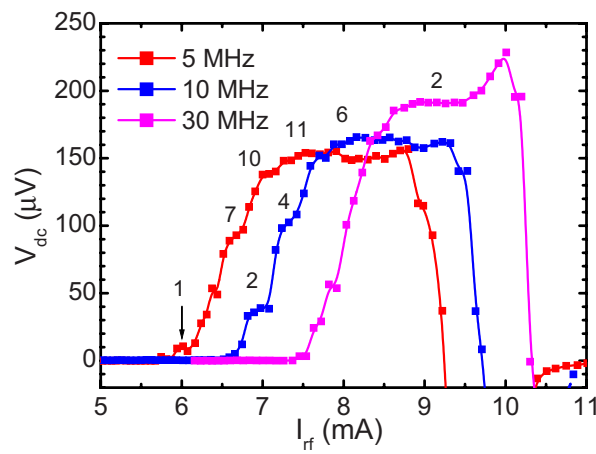

FIG. 8. (Color online) The $V_{\mathrm{dc}}$ dependence on the input rf current at 5, 10 and $30 \mathrm{MHz}$. The phase locking voltage steps are clearly observed (see the vertical arrows).

trapped vortex system, the sign reversal can be easily understood by considering either the inverted ratchet effect of interstitial vortices or the dynamical vortex reordering in the system. ${ }^{17,26}$ Obviously these mechanisms cannot properly account for our present observations. The most important difference from what was previously reported ${ }^{17}$ is that we find the sign reversal in the extremely high-frequency region ( $>=\mathrm{MHz}$ ). In the extremely high-frequency region the process of vortex motion being accelerated can dominate the behavior in the fast ratchet system, and this process is related to the inertial term. Therefore, we argue that the sign reversal in the present fast ratchet system should occur in the nonadiabatic state and the inertial term plays an important role in the behavior revealed in our measurements.

\section{Collective step-motor behavior}

Figure 8 presents our experimental results at 5, 10, and 30 MHz. A striking feature is the appearance of $V_{\mathrm{dc}}$ steps indicated by arrows (the data points are connected by lines for clarity. For the same reason only positive parts of $V_{\mathrm{dc}}$ are displayed in the figure). Such $V_{\mathrm{dc}}$ step implies usually that the vortex motion is locked to the external driver; i.e., the time for a vortex moving from one pinning center to another is commensurate with the period of the driver. ${ }^{3,13}$ In this case, the dc voltage step $V_{\mathrm{dc}}(n)$ is $2.76 \mathrm{nf}$ as explained in the introduction. The voltage step at $30 \mathrm{MHz}$ should be multiples of $82.5 \mu \mathrm{V}$. Our measured step value of $190 \mu \mathrm{V}$ corresponds approximately to $n=2$, i.e., the net displacement of vortex lattice in one cycle is $2 d_{0} . V_{\mathrm{dc}}$ as a function of the applied rf current measured at 5 and $10 \mathrm{MHz}$ are also displayed in Fig. 8. At $10 \mathrm{MHz}$, three voltage steps are clearly observed at 40, 102, and $165 \mu \mathrm{V}$, which correspond approximately to the values for even number steps. At $5 \mathrm{MHz}$, we can observe four steps at 12.8, 97.0, 128.4, and $152 \mu \mathrm{V}$, corresponding to $1 \mathrm{st}, 7 \mathrm{th}, 10 \mathrm{th}$, and 11 th steps. This analysis demonstrates that we have indeed observed a collective stepmotor behavior of the vortex lattice, i.e., approximately a quarter of a million single-quantum vortices in a quasi-2D array move synchronously the same distance. This collective rectification with interacting objects is different from the recent demonstration of a high-frequency ratchet in a threejunction superconducting quantum interference device loop, 


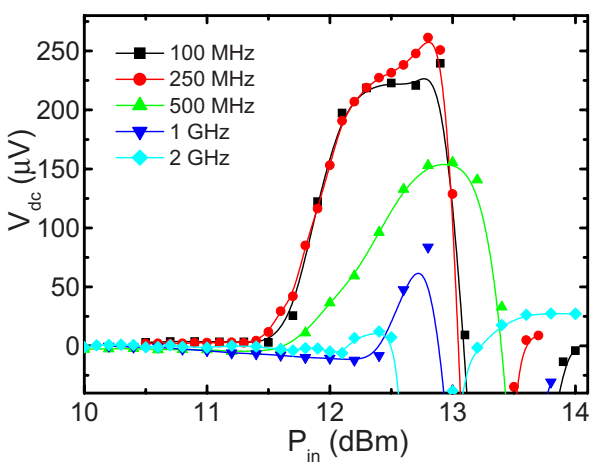

FIG. 9. (Color online) The rectified dc voltage $V_{\mathrm{dc}}$ (positive parts)dependence on the input power at 100, 250, $500 \mathrm{MHz}, 1$, and $2 \mathrm{GHz}$. The ratchet effect becomes weak in $\mathrm{GHz}$ region.

which behaves essentially like a single particle moving in a one-dimensional ratchet potential..$^{32,33}$ The demonstration of the collective behavior is of particular technological interest since the final rectification result is independent of the different initial conditions, for example, the separation of particles can be carried out for each particle starting with different initial conditions. ${ }^{34-36}$ Moreover, this collective behavior is also helpful in understanding the transportation behavior of the molecular motors inside the cell. ${ }^{27,28}$

However, the above demonstration is not perfect. For instance, we do not observed successive and equidistant dc voltage steps. The $V_{\mathrm{dc}}$ values for several steps do not agree strictly with the theoretical prediction. ${ }^{3,13}$ Furthermore, the number of the steps at $5 \mathrm{MHz}$ is not twice as many as that at $10 \mathrm{MHz}$, and the height is not about half of the corresponding value at $10 \mathrm{MHz}{ }^{3,13}$ This may be caused by the inhomogeneity of pinning potential in the film. At low frequencies, the vortices move a distance with a large number of $d_{0}$. Then, the influence on $V_{\mathrm{dc}}$ caused by some defects existing might be minor. However, at high frequencies, such influence will be enhanced because the vortices move only one or several $d_{0}$. We conclude more work should be done in order to get perfect $V_{\mathrm{dc}}$ step structure resulting from the phase locking in our vortex ratchet system.

\section{Vortex rectification at $\mathbf{G H z}$}

Figure 9 shows the $V_{\mathrm{dc}}$ dependence on the input power at the frequency of $100,250,500 \mathrm{MHz}, 1$, and $2 \mathrm{GHz}$. As the frequency is beyond $100 \mathrm{MHz}$, the resonance effect along the coaxial cable for rf power transmission becomes pronounced. So, the $\mathrm{rf}$ circuit is a distributed-parameter network, we had to use input power to represent the driving forces, instead of the rf current. ${ }^{37}$ It should be noticed that
$V_{\mathrm{dc}}$ is obtained by voltage readings at $B_{1}$ subtracting that at zero field. We found that $V_{\mathrm{dc}}$ with a pronounced plateau feature increases with the frequency and gradually disappears as the frequency is above $500 \mathrm{MHz}$, while the plateau feature disappears. The plateau feature, which is also observed at several ten $\mathrm{MHz}$ may correspond to the collective vortex lattice motion. However, the maximum $V_{\mathrm{dc}}$ decreases with the further increase of the frequency and the ratchet effect becomes very weak at $2 \mathrm{GHz}$ without any pronounced plateau features in $V_{\mathrm{dc}}$. The weak effect at $2 \mathrm{GHz}$ can be contributed to the limitation of the vortex motion driven by the fast alternating current, and another possibility may result from the unusual vortex motion in the disfigurement rows of the pinning lattices, which agrees with the previous measurement on the microstrip resonator. ${ }^{38}$

\section{CONCLUSIONS}

The fast vortex ratchet with the frequency varying from $0.5 \mathrm{MHz}$ to $2 \mathrm{GHz}$ is successfully observed in nanostructured asymmetric pinning system in superconducting thin film. We have found that transition from adiabatic (lowfrequency) to nonadiabatic (high-frequency) cases appears at about $1 \mathrm{MHz}$. In the high-frequency region, some new physical features are revealed, which are quite different from those in the low-frequency region. For example, the upward shift of ratchet window increases with the frequency, and the collective step-motor behavior and weak ratchet effect appears at GHz. In addition, we also find a sudden reversal of $V_{\mathrm{dc}}$ at large rf current, which could be explained using underdamped model. Above all, we conclude that our asymmetric pinning system with repulsive interaction vortices is effective in investigating the ratchet effect in high-frequency region and underdamped case.

\section{ACKNOWLEDGMENTS}

We acknowledge the support from the Research Fund K. U. Leuven Grant No. GOA/2004/02, Methusalem Funding by the Flemish Government and FWO Programmes. B.B.J. was supported by the FWO and K. U. Leuven Foundation and MOST 973 project (Project No. 2007CB31404). B.Y.Z. acknowledges the support from the MOST 973 project (Projects No. 2006CB601006 and No. 2009CB930803), the National Natural Science Foundation and the BIL FlandersChina Project of China. C.C.S.S. acknowledges support from the Brazilian agencies CNPq and FACEPE. We acknowledge $\mathrm{J}$. Van de Vondel to afford high quality $\mathrm{Pb}$ thin films. We acknowledge helpful discussions with T. Dahm and M. V. Fistul as well as the technical supports from R. Kutzner, Dr. E. H. Hollmann, Q. H. Chen, and W. Vinckx.
${ }^{1}$ R. P. Feynman, R. B. Leighton, and M. Sands, The Feynman Lectures on Physics (Addison-Wesley, Reading, MA, 1966), Ch. 46.

${ }^{2}$ P. Hanggi and R. Bartussek, Lecture Notes in Physics (Springer,
Berlin, 1996), Vol. 476, pp. 294-308.

${ }^{3}$ R. Bartussek, P. Hanggi, and J. G. Kissner, Europhys. Lett. 28, 459 (1994).

${ }^{4}$ See review article P. Reimann, Phys. Rep. 361, 57 (2002). 
${ }^{5}$ P. Hanggi, F. Marchesoni, and F. Nori, Ann. Phys. 14, 51 (2005).

${ }^{6}$ C.-S. Lee, B. Jankó, I. Derényi, and A.-L. Barabási, Nature (London) 400, 337 (1999).

${ }^{7}$ Q. Lu, C. J. O. Reichhardt, and C. Reichhardt, Phys. Rev. B 75, 054502 (2007).

${ }^{8}$ J. F. Wambaugh, C. Reichhardt, C. J. Olson, F. Marchesoni, and Franco Nori, Phys. Rev. Lett. 83, 5106 (1999).

${ }^{9}$ C. J. Olson, C. Reichhardt, B. Jankó, and F. Nori, Phys. Rev. Lett. 87, 177002 (2001).

${ }^{10}$ B. Y. Zhu, F. Marchesoni, and F. Nori, Physica E 18, 318 (2003).

${ }^{11}$ B. Y. Zhu, F. Marchesoni, and F. Nori, Phys. Rev. Lett. 92, 180602 (2004).

${ }^{12}$ B. Y. Zhu, L. Van Look, V. V. Moshchalkov, B. R. Zhao, and Z. X. Zhao, Phys. Rev. B 64, 012504 (2001).

${ }^{13}$ B. Y. Zhu, F. Marchesoni, V. V. Moshchalkov, and F. Nori, Phys. Rev. B 68, 014514 (2003).

${ }^{14}$ R. Wördenweber, P. Dymashevski, and V. R. Misko, Phys. Rev. B 69, 184504 (2004).

${ }^{15}$ J. Van de Vondel, C. C. de Souza Silva, B. Y. Zhu, M. Morelle, and V. V. Moshchalkov, Phys. Rev. Lett. 94, 057003 (2005).

${ }^{16}$ C. C. de Souza Silva, J. Van de Vondel, B. Y. Zhu, M. Morelle, and V. V. Moshchalkov, Phys. Rev. B 73, 014507 (2006).

${ }^{17}$ C. C. de Souza Silva, J. Van de Vondel, M. Morelle, and Victor V. Moshchalkov, Nature (London) 440, 651 (2006).

${ }^{18}$ J. E. Villegas, S. Savel'ev, F. Nori, E. M. Gonzalez, J. V. Anguita, R. Garcia, and J. L. Vicent, Science 302, 1188 (2003).

${ }^{19}$ L. Dinis, E. M. González, J. V. Anguita, J. M. R. Parrondo, and J. L. Vicent, Phys. Rev. B 76, 212507 (2007).

${ }^{20}$ W. Gillijns, A. V. Silhanek, V. V. Moshchalkov, C. J. O. Reichhardt, and C. Reichhardt, Phys. Rev. Lett. 99, 247002 (2007).

${ }^{21}$ F. Nori, Nat. Phys. 2, 227 (2006).

${ }^{22}$ Y. Togawa, K. Harada, T. Akashi, H. Kasai, T. Matsuda, F. Nori, A. Maeda, and A. Tonomura, Phys. Rev. Lett. 95, 087002
(2005).

${ }^{23}$ H. B. Wang, B. Y. Zhu, C. Gürlich, M. Ruoff, S. Kim, T. Hatano, B. R. Zhao, Z. X. Zhao, E. Goldobin, D. Koelle, and R. Kleiner, Phys. Rev. B 80, 224507 (2009).

${ }^{24}$ D. Cole, S. J. Bending, S. Savel'ev, A. Grigorenko, T. Tamegai, and F. Nori, Nat. Mater. 5, 305 (2006).

${ }^{25}$ S. Ooi, S. Savel'ev, M. B. Gaifullin, T. Mochiku, K. Hirata, and F. Nori, Phys. Rev. Lett. 99, 207003 (2007).

${ }^{26}$ D. E. Shalom and H. Pastoriza, Phys. Rev. Lett. 94, 177001 (2005).

${ }^{27}$ F. Jülicher and J. Prost, Phys. Rev. Lett. 75, 2618 (1995); 78, 4510 (1997).

${ }^{28}$ M. Badoual, F. Jülich, and J. Prost, Proc. Natl. Acad. Sci. U.S.A. 99, 6696 (2002).

${ }^{29}$ A. V. Silhanek, L. Van Look, S. Raedts, R. Jonckheere, and V. V. Moshchalkov, Phys. Rev. B 68, 214504 (2003).

${ }^{30}$ P. Jung, J. G. Kissner, and P. Hanggi, Phys. Rev. Lett. 76, 3436 (1996).

${ }^{31}$ J. Mateos, Phys. Rev. Lett. 84, 258 (2000).

${ }^{32}$ I. Zapata, R. Bartussek, F. Sols, and P. Hanggi, Phys. Rev. Lett. 77, 2292 (1996).

${ }^{33}$ A. Sterck, R. Kleiner, and D. Koelle, Phys. Rev. Lett. 95, 177006 (2005).

${ }^{34}$ C. Kural, H. Kim, S. Syed, G. Goshima, V. I. Gelfand, and P. R. Selvin, Science 308, 1469 (2005).

${ }^{35}$ J. Rousselet, L. Salome, A. Ajdari, and J. Prost, Nature (London) 370, 446 (1994).

${ }^{36}$ A. van Oudenaarden and S. G. Boxer, Science 285, 1046 (1999).

${ }^{37}$ D. M. Pozar, Microwave Engineering (John Wiley \& Sons, Inc., New York, 1998).

${ }^{38}$ High Temperature Superconductivity 2, edited by A. V. Narlikar (Springer Verlag, Berlin-Heidelberg, 2004), p. 363-411. 